\title{
Cold thread Forming - the chipless alternative for high resistant internal threads
}

\author{
Roland Heiler* \\ HTW - Berlin, Department of mechanical engineering, 12459 Berlin, Germany
}

\begin{abstract}
In the area of construction and mechanical engineering the designers and production engineers very often do have the task to produce high resistant connections with the possibility of assembling and de assembling parts in a wide range of different materials. Most often connection bolts and internal threads are used to fulfill this task. To produce the internal threads typically the three following internal threading technologies are used: tapping, cold thread forming and thread milling. Tapping and thread milling are metal cutting technologies but cold thread forming is a chipless alternative. Due to the cold forming of the thread this process does have several benefits in comparison to the traditional cutting technologies, but also some special features and process characteristics have to be considered, that it is possible to produce reliable internal threads. In the article the technology is presented with information about the process characteristics, the modern tools design and typical application areas. Results of pull out tests are showing the possibility for producing high resistant threads.
\end{abstract}

\section{Technologies to produce internal threads}

In the area of construction and mechanical engineering connecting parts together is a typical and often found task. Very often for a stable construction welding technologies are used. But this connection is not possible to de assemble if it is necessary to change parts or components. If it is necessary to change parts of the whole construction a connection with the possibility of a de assembling without destroying one of the parts is normally recommended. In this case, the design engineer will normally use a connection consisting of internal thread and bolt. Due to the well know international standards, this thread bolt connections can be found in nearly all construction and industry areas, material components and a wide range of dimensions. Also depending on the application area different thread profiles are used also in metric or inch dimensions.

The connection is based on a bolt with the necessary external thread profile and a internal thread for the fastening. Most often the standardized external thread bolts are produced by a cold or hot forming process. For special dimensions and mainly not standardized applications turning is also a common technology for producing the external threads. To produce the internal threads three different technologies are today typically used in the industry: thread tapping, thread milling and cold thread forming. Tapping and

\footnotetext{
* Corresponding author: roland.heiler@htw-berlin.de
} 
thread milling are cutting technologies, while thread forming is a cold forming process. Each technology does have some special benefits for the usage and application areas. Tapping e.g. is widely used in the industry, because it is well known, standard or even special taps are supplied from the precision tool manufacturing industry for a wide range of applications and it is possible to produce internal thread into the most common materials like cast iron, aluminium, steel or even hardened steel. The technology of thread milling was used in the past mostly for big thread dimensions above the common range of tapping. Here we do have a lower torque during the manufacturing operation because it's a milling process. Today thread milling will be used also in the same application range like conventional tapping. Due to the milling operation and the helical interpolation of the three necessary axes to produce the internal thread, thread milling is most often slower in the producing cycle in comparison to conventional tapping. Due to the high process reliability, even if we do have a tool breakage the workpiece will not be destroyed, because it is possible to complete the internal thread with a new tool, thread milling is often used when producing expensive workpieces where a failure would cause a cost intensive damage. In comparison to the conventional tapping process, cold thread forming is less known, even if this technology does have some significant benefits in comparison to the standard tapping technology. But also some special requirements are necessary to observe, when the cold thread forming should be used in the production or replace the standard tapping process.

\section{The cold forming process}

In the first step to produce the internal thread it is necessary to produce the pre hole in the material. Beside the dimension of the thread (diameter of the thread, tolerance of the thread and pitch size) the hole diameter is also depending on the used technology for the threading operation. When tapping is used, the necessary material for the thread is removed by the tap. In the cold forming operation the cold forming tap is screwed into the material and the thread profile is produced by deforming the material. The material is flowing axial and radial on the flank of the cold forming tap and a characteristic profile is produced. During the conventional tapping process the internal thread diameter was already produced by the drilling operation, whereas the internal thread diameter with the cold forming process is only produced during the material deformation. Due to this effect, the drill diameter for conventional tapping operations is standardized, while the hole diameter for the cold forming operation is mainly depending on the machined material, the conditions of friction on the flank of the cold forming tap, the flank tolerance of the tool and the forming speed. In comparison to the conventional tapping the diameter of the drill has to be bigger, because the internal thread diameter is produced by deforming the material. If the same drill size would be used for conventional tapping and cold forming, too much material has to be deformed from the forming tap. This would cause a high increase of the necessary torque during the forming operation and in the last stage of the operation a jamming of the tool in the produced thread. Tool and internal thread would be destroyed by cold welding. 


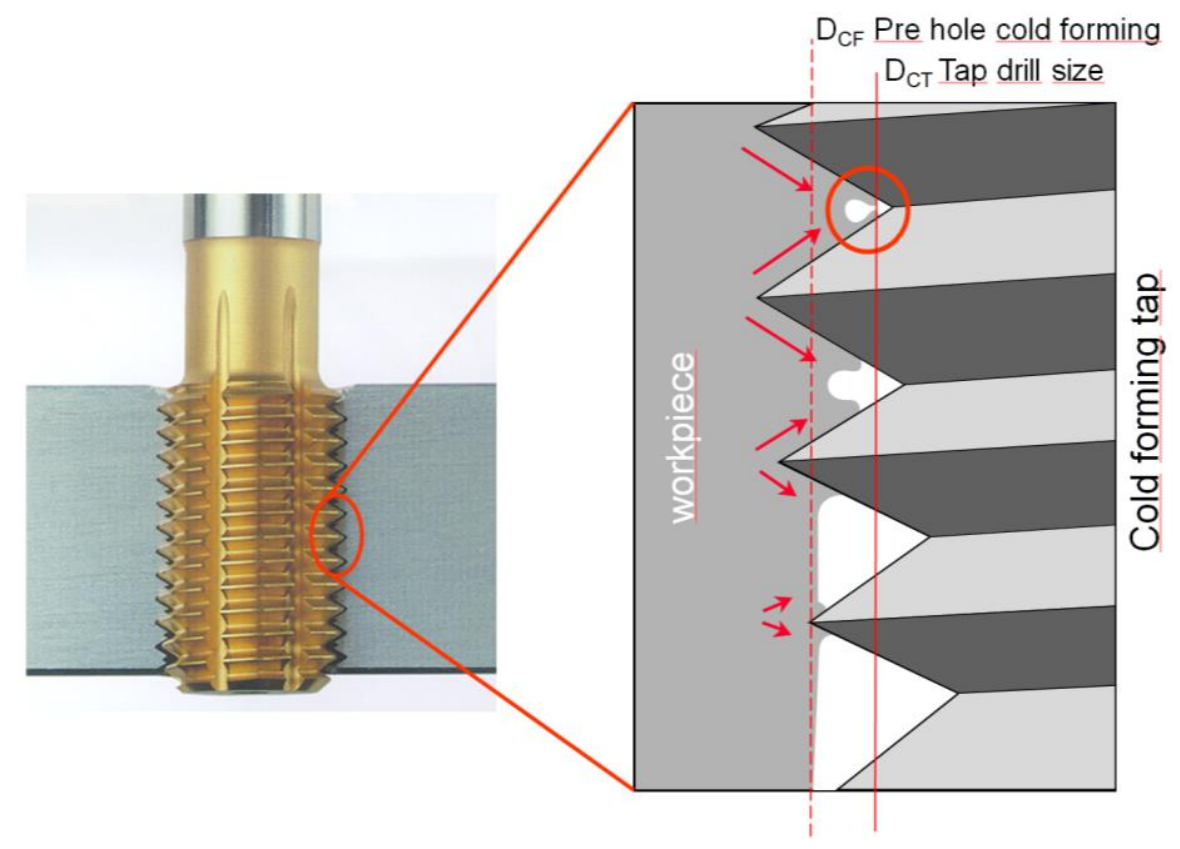

Fig. 2: Cold forming process and tool [in accordance to 1] with drill sizes and material deformation

The necessary drill diameter for a conventional tapping process (DСT) can be calculated by the following formula for a metric thread.

$$
\text { Tap drill size } \text { Dст }=\text { Thread diameter }- \text { pitch size }
$$

For the cold forming process the drill diameter $\left(\mathrm{D}_{\mathrm{CF}}\right)$ must be bigger. For a first step in the production planning the recommended drill diameter can be calculated with the following formula

Pre hole cold forming $D_{\mathrm{CF}}=$ Thread diameter $-0.45 \mathrm{x}$ pitch size

With an increase of the drill diameter, less material will be deformed by the cold forming tool and a reduction of the necessary torque during the operation is possible. On the other side, the resulting inner diameter of the thread will be bigger and the overlapping of the flanks from internal and external thread (bolt flank) will be reduced, which causes a decline of the thread strength. For serial or mass production of internal threads a application wise detail view of the drill size is recommended.

\section{Material and application properties}

Conventional tapping and thread milling is possible to use in all machinable materials. For cold thread forming this is one of the main limitations. It is only possible to use cold thread forming, if the material is formable. To evaluate if the technology of cold thread forming is possible to use in the application three material characteristics should be analyzed: the elongation rate, the residual strength and the content of silicon in Aluminum-Silicon alloys.

The elongation rate, typically the A5 factor of the material, should be above $5-10 \%$. Below 5\% elongation rate it is still possible to screw in the cold forming tap, but the 
material is not transformed into the typical thread profile and the inner diameter of the thread is not reduced. In this case the cold forming tap is machining or cutting the material.

Beside the elongation rate, the residual strength of the material is the next important factor. Today it is possible to use the cold forming operation in materials with a residual strength up to $1000 \mathrm{MPa}$. In some publications even $1400 \mathrm{MPa}$ as a maximum residual strength of the material can be found. Above $1400 \mathrm{MPa}$ the material it is not possible or recommended to use the cold forming process. Even above $1000 \mathrm{MPa}$ a significant reduction of the tool life can be found.

For Aluminum-Silicon alloys the content of silicon is the determining parameter. Aluminum-Silicon alloys with a content of Silicon above $12 \%$ are not recommended for the process. In hypereutectic Aluminum-Silicon alloys, we can find free Silicon structures in the material matrix. During the cold forming process it is possible to deformed the material matrix, but it is not possible to deform the free Silicon structure, with its brittle behavior. These parts of the material will break out of the structure and will results in a very uneven surface structure as well as a lot of micro cracks in the thread.

Due to these material properties, the cold thread forming is most often used in soft, ductile materials or Aluminum-Silicon alloys with a relatively low content of Silicon. Typical materials are e.g. construction steel, stainless austenitic steel, soft copper alloys and Aluminum-Silicon alloys. The usage in the area of nodular cast iron with a high elongation rate could be possible, but residual strength tests of the threads are recommended as well as a micro analysis of the thread structure. It was also possible to produce internal threads with a high resistant strength in Titanium alloys grade 5 (TiAl6V4). The tests were made with relatively small threads (M3), but show the principle possibility to use the cold forming technology also in this material. For other dimensions and real applications in Titaniumalloys further tests are recommended [2].

Due to the low elongation rate, cold thread forming is not possible or recommended to use in brittle, short chipping, materials like grey cast iron, hardened steel, magnesium alloys or short chipping brass.

Beside the material properties, also the application area should be analyzed. The inner diameter of the thread is created by the deforming of the material and results in the typical crater like profile of the thread. In contrast to the forming process, the inner thread diameter is produced by the drill diameter when using conventional cutting technologies for the threading, like tapping or thread milling. The crater profile of cold formed threads can cause problems in some applications like the food industry, medical industry and aerospace, turbine or hydraulic applications. In the food industry the components have to be cleaned very often and no food or material components are allowed to leave behind in the thread. With the crater profile this is not $100 \%$ excluded. The same problem is seen in medical applications, were infections could be a problem. In hydraulic or turbine components, smallest parts could cause major problems for the whole application. Also in these areas the usage of cold thread profiles must be checked before more in detail, if cold formed threads are possible to use.

From the application point of view, it is possible to use the same tool for blind hole or through hole threads. It is not necessary to design a special chamfer geometry for both application.

\section{Tool geometry and coating}

On the first, rough view, conventional cutting taps and cold forming taps seems to be quite similar, due to the typical thread profile of the tools. But on a closer look, more in detail, both tool types are totally different. The characteristic feature of the conventional cutting taps, is the cutting geometry of the tools. For this the tools do have a chamfer part and 
grinded cutting flutes. This basic geometry is necessary, to produce the internal thread by removing the material while cutting the thread. In opposite to this, cold forming tools don't need or have cutting flutes, because the thread profile is produced by the deforming material. Their geometry is more similar to a conventional "round" bolt. But to deform the material, the cross section of the cold forming taps is not "round", but has a "polygon profile". The characteristic polygon profile guaranties a smooth deformation of the material and a reduced friction during the forming operation. Also it enables the lubrication fluid or oil to reach the flank of the tool, which results in a further significant reduction of the friction. To improve the transportation of the lubrication during the process, it is possible to produce small lubrication grooves (lubrication flutes) in the "valley of the polygon profile" or even to use tools with an internal lubrication supply. Due to the bigger cross section of the cold forming tap, even with small lubrication flutes, the forming taps do have a high stability and the risk of a tool breakage during the production is reduced. During conventional tapping operations, especially in blind holes and long chipping materials, the produced chips can cause chip transportation problems, chip jamming and tool breakage. Because there are no chips produced during cold thread forming, this technology has also a higher process reliability. Cold thread forming taps are produced with polygon profiles for universal usage in standard production applications as well as specially designed profiles for special material properties. Here we can find tool designs for very soft materials like wrought aluminum, austenitic stainless steel materials as well as high strength steel material. For each application the polygon profile can be optimized to reduce friction and torque during the tapping [3]. Beside the geometry these tools are also coated with thin multilayer coatings based on e.g. Titanium-Nitride, Titanium-Aluminum-Nitride or TitanCarbon-Nitride. With the coating it is possible to reduce the friction during the operation, as well as increase significantly the surface hardness. Through both effects it is possible to increase the tool life. It is possible to use tools with a bright finish surface or a ChromiumNitride surface especially for materials with a strong tendency of cold welding effects like e.g. soft, wrought aluminum alloys. The length of the chamfer part is standardized like for conventional cutting taps. Most often Form C (length $2-3$ pitch) or Form E (1.5 - 2 pitch) can be found in the industry.

\section{Thread properties and application benefits}

In comparison to conventional threads, cold formed threads do have a significant different material structure. In rolled material, the material structure (so called "material fibers") are normally not destroyed but only deformed and banded. Due to the cold forming process, depending on the material properties, it is possible to determine an increase of the material hardness. Also the surface structure is smooth in comparison to conventional produced threads. These effects, bended "fibers", cold work hardening and a smooth surface structure are positive aspects for the classification of cold formed threads and resulting in high resistant threads. In comparison to conventional threads it is possible to increase the resistant strength of the threads. Especially for threads with a reduced quantity of pitch, it is possible to show the higher strength on cold formed threads [4]. But it should be mentioned, that normally above a thread depth of 1.5 times the diameter of the thread, this advantage is not possible to see in the tests. 


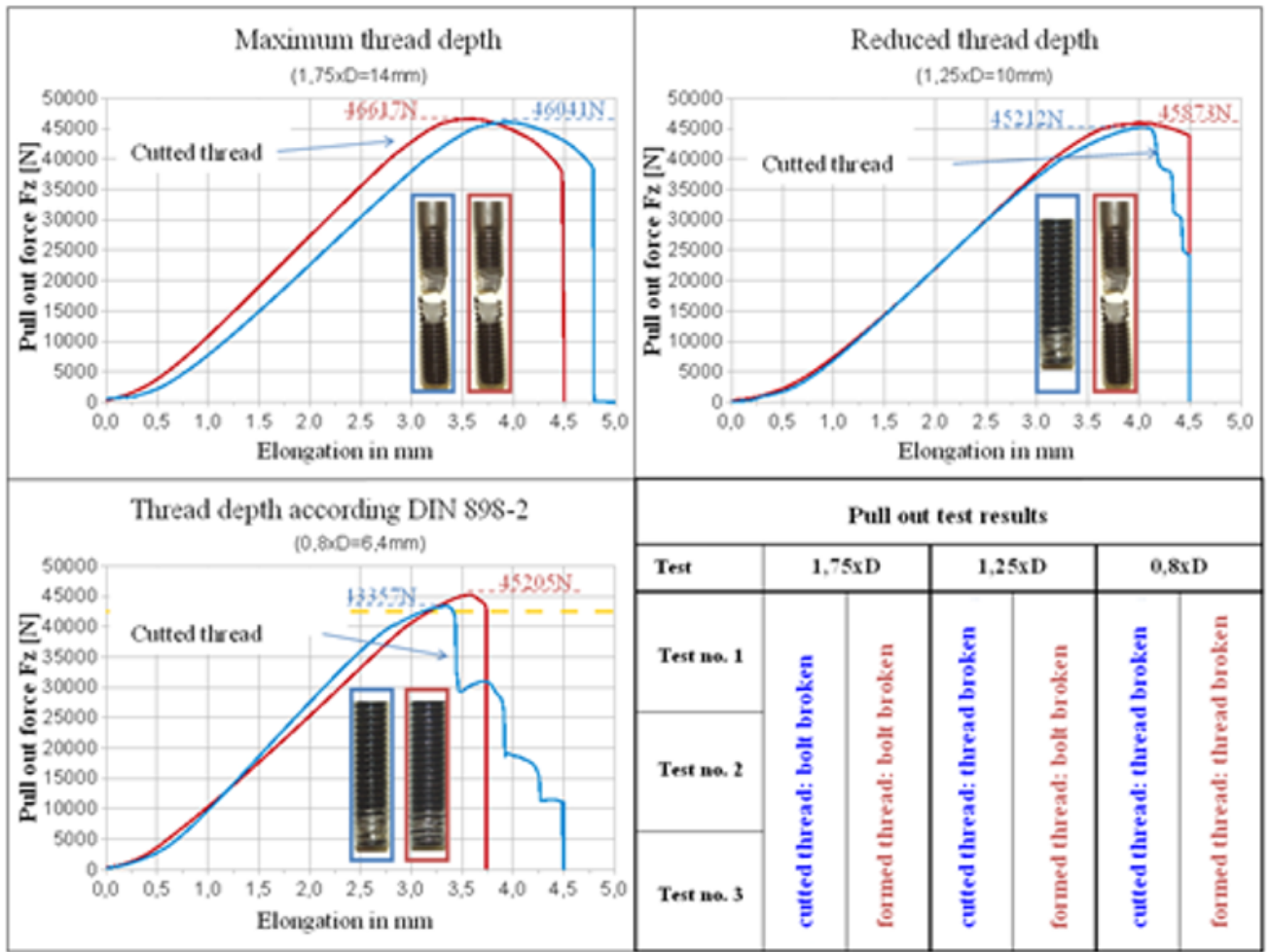

Fig. 2: pull out resistance in comparison conventional machined threads and cold formed threads [4]

Beside the high thread strength, other aspects are even more interesting to show for the cold thread forming process. As mentioned above, it is possible to reduce the tool variety in the production, because one cold forming tap is possible to use in through as well as blind hole production. Also it is possible to use one tool for all materials (if these materials are suitable for cold forming), if the tool life and productivity is not the highest goal in the production. If productivity and tool life are the most important issues, special design forming lubes and coatings can be supplied. Due to the rounded, often polished, forming geometry of the tools in combination with a multilayer hard coating, the tool life is in comparison to conventional cutting taps higher. Especially in long chipping, ductile and jamming materials like e.g. austenitic stainless steel, the cold forming process does have a higher process reliability than the conventional cutting tap, because the thread is formed and no chips can cause any problem. This is even more important in deep blind holes (above 2 times the diameter). The cold forming tap is guided by itself in the resulting thread, oversized threads are not possible. Together with the higher stability of the tool, a main benefit of the technology is the high process reliability. The forming speed and production time, when enough lubrication is used, can be the same or even higher, compared to conventional bright finish cutting taps or taps with only a steam oxide surface. For serial or mass production with new, stabile machine tools and tool attachments, it is also possible to use solid carbide cold forming taps. In comparison to tools made of high speed steel and depending on the machining conditions, the tool life can be much higher. 


\section{Summery}

Cold thread forming is a chipless alternative in producing high strength internal threads. Especially material properties and some special application requirements have to be considered. Cold thread forming can be used in ductile materials with a minimum elongation rate of A5 5\% and a maximum tensile strength of ca. $1000 \mathrm{MPa}$ (in some article $1400 \mathrm{MPa}$ under special lubrication conditions). For Aluminum-Silicon-Alloys a maximum content of $12 \%$ Silicon is possible. The produced threads are characterized by a high resistant strength due to cold hardening and uninterrupted material structures (depending on the material properties). The threads are also characterized by the typical crater profile in the inner diameter of the thread, which must be observed for the selected application. The tools do have a high stability and guarantee excellent process reliability with a long tool life and high productivity in production. The technology can be used in construction engineering, automotive industry and suppliers, as well as general engineering applications, where high resistant threads for a detachable connection are required.

\section{References}

1. F. Tikal, R. Heiler, P. Müller, Bohrer und Gewindewerkzeuge, Werkzeugkonzepte, Fertigungstechnologien und elektronische Werkzeugauswahl. Die Bibliothek der Technik, Band 257, Verlag Moderne Industrie 2003

2. R. Heiler, P. Tischkau, Hochleistungsgewindefertigung in Titanlegierungen. Forschungsprojekt Hochschulbasierte Weiterbildung für Betriebe an der HTW-Berlin, 2015

3. M. Schäfer, Analyse und Beschreibung des Innengewindefertigungsverfahrens Gewindefurchen auf Basis eines Modellversuchs (Dissertation, Universität Gesamthochschule Kassel, 2004)

4. Cordes, Oliver, Untersuchungen zur Innengewindefertigung in hitzebeständigem Edelstahlguss für Turboladergehäuse. Master-thesis, HTW-Berlin 2012 